\title{
Erratum to: Chaos Detection and Predictability
}

\author{
Charalampos (Haris) Skokos, Georg A. Gottwald, Jacques Laskar
}

Erratum to:

Ch. Skokos et al. (eds.), Chaos Detection and Predictability, Lecture Notes in Physics 915, DOI 10.1007/978-3-662-48410-4

(c) Springer-Verlag Berlin Heidelberg 2016

The Editor name "Charalampos Haris Skokos" was not correct. It is now corrected to Charalampos (Haris) Skokos throughout the book.

The publisher apologizes for having published an early draft edition of the preface. This has been updated to the final version.

The updated original online version for this book can be found at DOI 10.1007/978-3-662-48410-4

Ch. (Haris) Skokos

Department of Mathematics and Applied Mathematics, University of Cape Town, Rondebosch, South Africa

e-mail: haris.skokos@uct.ac.za

G.A. Gottwald

School of Mathematics and Statistics, University of Sydney, Sydney, Australia

J. Laskar

Observatoire de Paris, IMCCE, Paris, France

(C) Springer-Verlag Berlin Heidelberg 2016 